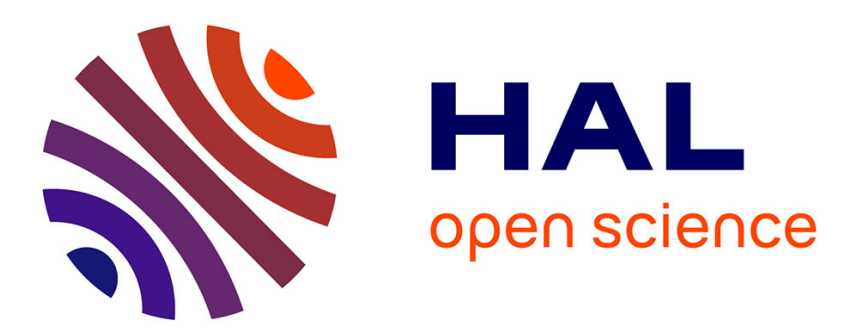

\title{
La prova della soglia: migranti africani tra mobilità e immobilizzazione
}

Philippe Poutignat, Jocelyne Streiff-Fénart

\section{To cite this version:}

Philippe Poutignat, Jocelyne Streiff-Fénart. La prova della soglia: migranti africani tra mobilità e immobilizzazione. Mondi Migranti (Rivista di studi e ricerche sulle migrazioni internazionali), 2016, 1. halshs-01332453

\section{HAL Id: halshs-01332453 https://shs.hal.science/halshs-01332453}

Submitted on 15 Jun 2016

HAL is a multi-disciplinary open access archive for the deposit and dissemination of scientific research documents, whether they are published or not. The documents may come from teaching and research institutions in France or abroad, or from public or private research centers.
L'archive ouverte pluridisciplinaire HAL, est destinée au dépôt et à la diffusion de documents scientifiques de niveau recherche, publiés ou non, émanant des établissements d'enseignement et de recherche français ou étrangers, des laboratoires publics ou privés. 
La prova della soglia: migranti africani tra mobilità e immobilizzazione

Philippe Poutignat

URMIS Université de Nice Sophia Antipolis/Université Paris Diderot/CNRS/IRD

e Jocelyne Streiff-Fénart

URMIS Université de Nice Sophia Antipolis/Université Paris Diderot/CNRS/IRD

\begin{abstract}
s :
Questo articolo verte sulle migrazioni africane che vengono définite irregolari o clandestine. La principale caratteristica di queste migrazioni è infatti quella di essere una forma di mobilità ostacolata. Ponendo il controllo della frontiera al cuore della questione migratoria le istituzioni preposte al controllo migratorio hanno al tempo stesso spostato il senso che per gli stessi individui migranti riveste la mobilità, ormai sentita come una prova di superamento. Il nostro interesse si è portato sull'esperienza sociale di quei migranti la cui vita si svolge sotto la costrizione dell'immobilizzazione.

This article discusses African migrations known as irregular or clandestine. The main characteristic of these migrations is that they represent a mobility hindered by the closure of Europe's borders and the continuous hardening of the policies meant to repulse the people crossing. By placing the control of the border at the heart of the question of migrations, the institutions in charge of migratory control have influenced the meaning that the people migrating have of mobility. It is now apprehended as the challenge of the threshold. We are interested in particular by the social experience of these migrants whose life unfolds under the constraint of immobility.
\end{abstract}

Clandestini, migranti africani, controllo migratorio

Questo articolo verte sulle migrazioni africane che vengono définite irregolari o clandestine ${ }^{1}$. Tali migrazioni vengono usualmente affrontate da un punto di vista eurocentrico che si preoccupa soprattutto del loro punto di arrivo: la presenza di questi migranti clandestini nei paesi europei. Qui noi li considereremo nel loro punto di partenza in Africa, che per molti di loro si trova essere uno spazio di immobilizzazione forzata: lo spazio nel quale questi migranti circolano da un paese africano all'altro, alla ricerca di un passaggio a destinazione dell'Europa. La principale caratteristica di queste migrazioni è infatti quella di essere una forma di mobilità ostacolata, "contrariata" (Le Houérou 2007) dalla chiusura delle frontiere europee e dal continuo rafforzamento delle misure che si propongono di respingere i candidati al passaggio.

\footnotetext{
${ }^{1}$ Poggia su osservazioni fatte con regolarità nelle città di Nouadhibou in Mauritania e di Bamako nel Mali fra il 2005 e il 2009 nel contesto di un programma di ricerca collettivo (MITRANS) finanziato dalla Agence Nationale pour la Recherche.
} 
A partire dal summit di Siviglia nel 2002 le istituzioni europee hanno messo in pratica una politica di lotta contro la migrazione clandestina in due parti:

- il dispiego di un dispositivo di sorveglianza delle frontiere terrestri e marittime: messa in piedi del Sistema Integrato di Sorveglianza Esterna (SIVE), nel distretto di Gibilterra (a poco a poco esteso fino alle Canarie), e nel 2004 la creazione dell'agenzia Frontex.

- una politica di partnership euro-africano (conferenza di Rabat nel 2006), completata da accordi bilaterali con gli Stati delle zone da cui provengono e dove transitano i migranti, per cercare di fermarli prima che oltrepassino una delle frontiere europee. Per quanto riguarda, per esempio, la frontiera spagnola si può menzionare il progetto Seahorse del 2005 (condotto dalla Spagna con l'appoggio finanziario del programma AENEAS $^{2}$ della commissione europea), poi in seguito il programma Atlantis, che implica assieme alla Guardia civil e al Ministero Spagnolo degli interni la Mauritania, il Marocco, il Senegal e il Capo Verde.

Una delle poste in gioco di una tale politica di partnership è, beninteso, l'ottenere un allineamento negoziato di questi stati cuscinetto sugli obbiettivi di lotta contro la migrazione irregolare. Si tratta innanzitutto di ottenere la loro firma su accordi di riammissione, ma ugualmente di far internalizzare questa politica dai paesi del Sud e di farla accettare alle loro opinioni pubbliche. L'idea che il controllo migratorio è un'impresa collettiva, che deve essere portata a termine in maniera concertata da partner cooperanti al fine di massimalizzare i loro vantaggi rispettivi, è diventata uno slogan dell' $\mathrm{IOM}^{3}$ : "Per il beneficio di tutti".

Il beneficio del controllo consisterebbe per gli stati del Nord nel proteggersi dall'afflusso dei migranti e dalle minacce che questo farebbe pesare sui mercati del lavoro e sui sistemi di protezione. In quanto agli stati del Sud, la buona volontà manifestata nella cooperazione nei dispositivi di controllo permetterebbe loro di guadagnare delle risorse finanziarie o degli impieghi per i loro cittadini espatriati (è ciò che gli attivisti denunciano come "il mercanteggiamento" degli scambi di riammissione), e accordi di cooperazione permetterebbero loro di utilizzare al meglio gli apporti di emigrati regolari (co-sviluppo).

È in questo contesto di esternalizzazione del controllo che alcuni paesi, in particolare quelli del Maghreb", sono diventati dei "paesi di transito": sono considerati come degli spazi frontiera, non in virtù di una linea di demarcazione che separi nettamente due territori, ma piuttosto per l'anticipazione di un virtuale oltrepassare una tale linea situata più a Nord.

\footnotetext{
${ }^{2}$ Programme for financial and technical assistance to third countries in the area of migration and asylum.

${ }^{3}$ International Organization for Migration. Per un'analisi del ruolo di quest'organismo inter-governativo, cfr. Georgi, 2010.

${ }^{4}$ Ma ugualmente tutti quelli che "accolgono" gli espulsi e i ricacciati o che fungono da tappa sulle vie terrestri verso le coste mediterranee o atlantiche, come il Mali o il Niger.
} 
Il termine qualificativo "paese di transito" mette in avanti visioni della migrazione edei migranti elaborate in funzione di poste migratorie proprie all'Europa (la gestione dei flussi migratori verso il suo territorio), che si sovrappongono alle rappresentazioni locali della mobilità e dei migranti e contribuiscono a modificarle. Cittadini di paesi della sub-regione che circolavano in maniera assolutamente legale nello spazio di circolazione della $\mathrm{CEDEAO}^{5}$, di cui sono membri la maggiorparte di questi paesi, vengono ormai interpellati come dei potenziali illegali e come dei clandestini, cosa che non necessariamente sono, ma che li si suppone poter diventare ulteriormente, quando avranno valicato le frontiere dell'Europa.

È dunque degno di nota che il problema della "migrazione illegale" al quale tentano di replicare le politiche migratorie dell'unione europea non si pone in maniera indipendente dalle "soluzioni" che queste stesse politiche pretendono dargli: non pre-esiste all'irrigidimento dei dispositivi di sorveglianza e di controllo, ma concorre a questi.

L'importanza estrema che ha preso questo dispositivo di controllo militarizzato e la risonanza mediatica di queste migrazioni clandestine vanno di pari passo col ricorrere di "crisi migratorie". Queste ultime sono l'occasione di una messa in piedi di nuovi mezzi repressivi che si presumono assicurare l'integrità delle frontiere. Simultaneamente contribuiscono, occupando le prime pagine della cronaca, a riattivare e ad attualizzare i dibattiti sulla politica migratoria ai diversi livelli nazionali, regionali ed europei. Tali crisi, dove si annunciano delle "invasioni" di migranti illegali che ogni volta sorpassano tutto quello che si era conosciuto fin qui, si iscrivono in una visione fondamentalmente negativa della migrazione in generale, percepita come anomala e irrazionale. La riduzione dei flussi è posta come un imperativo, partendo dal principio che per la gente è meglio restare a casa propria, mentre in molte regioni d'Africa, specie in Africa dell'Ovest, non si considera affatto anormale che la gente si muova: l'Africa ha un'antica e forte tradizione di mobilità, con migrazioni commerciali, religiose e anche con migrazioni di lavoro che attirano verso i poli d'attività agricole o industriali nei paesi vicini: le piantagioni di cacao in Costa d'Avorio, il bacino arachideo per delle migrazioni stagionali in Senegal, più recentemente il petrolio in Nigeria, in Algeria e in Libia, paese che contava più migranti sub-sahariani che qualsiasi paese d'Europa. Non c'è famiglia, tranne quelle più povere, che non conti uno o più migranti nei propri ranghi e la migrazione è piuttosto considerata una ricchezza, non soltanto in quanto fonte di guadagno grazie ai trasferimenti di soldi, ma anche in termini di prestigio.

Ponendo il controllo della frontiera al cuore della questione migratoria le istituzioni preposte al controllo migratorio hanno al tempo stesso spostato il senso che per gli stessi individui migranti riveste la mobilità, ormai sentita come una prova di superamento (Streiff-Fénart e Segatti, 2011): una prova nel senso di qualcosa di spossante (la violenza fatta a quei migranti,

\footnotetext{
${ }^{5}$ Comunità economica degli Stati Africani dell'Ovest. Organismo inter-governativo raggruppante 7 paesi dell'Africa dell'ovest.
} 
le sofferenze durante la traversata del deserto o le ferite durante i tentativi di oltrepassare le barriere, i naufragi), prova nel senso di esame, che si valuta in termini di successo o di fallimento, prova anche nel senso di qualcosa che si prova come un'esperienza di vita particolare di cui solo quelli che la vivono possono rendere conto.

Un'altra conseguenza di questa situazione è lo sviluppo di una forma di mobilità che non va da un punto ad un altro, ma lascia i migranti fuori dal loro punto di partenza in una situazione di transito all'interno del continente. Il nostro interesse di antropologi specialisti di migrazioni si è dunque portato sull'esperienza sociale di quei migranti la cui vita si svolge sotto la costrizione dell'immobilizzazione, bloccati in un luogo "Aspettando la felicità" come dice il regista maliano Abderrahmane Sissako, nel film che ha girato su questo tema, filmato a Nouadhibou nel 2002.

Nel corso della nostra indagine ci è apparso tuttavia che per capire in tutta la sua complessità questa esperienza sociale dei migranti in transito bisognava ricollegarla non soltanto agli effetti della militarizzazione del controllo, ma anche ad un processo situato ad un altro livello e ad un'altra profondità temporale: la relativa individualizzazione dei progetti di vita presso $i$ giovani d'Africa, che fa della migrazione un'avventura personale e una prova di realizzazione di sé (Timéra 2001).

Riguardo al primo aspetto, spicca l'impatto dei dispositivi finalizzati a ostacolare la mobilità sulle pratiche migratorie, che diventano de facto delle tattiche di sovversione (come il ricorso ai passatori, i falsi documenti, i cambi d'identità). Impatto anche sugli itinerari e sui percorsi della migrazione che sono divenuti più sinuosi e a denti di sega per adattarsi alle modalità del controllo con dei cambiamenti di rotta: apertura di nuove vie, al prezzo di rischi incrementati quali, un tempo, le partenze sempre più a sud che miravano a raggiungere le Canarie; ma anche cambio rapido degli itinerari preferiti, come nel caso del ribaltarsi delle strade verso l'Europa dall'ovest (la Spagna tramite Gibilterra o le Canarie) all'est (Libia, Tunisia); tutto ciò implicando soste prolungate in luoghi di tappa e in città di staffetta per "rifarsi" dopo le espulsioni e le cacciate e percercare una nuova soluzione per il passaggio. Queste pratiche migratorie sono imposte dall'esterno, dalle costrizioni che pesano sugli spostamenti; cionondimeno hanno logica ed effetti propri. Hanno generato forme specifiche d'occupazione dello spazio: degli accampamenti, delle foreste (come quella di Ben Younes presso l'enclave di Ceuta in Marocco), dei ghetti ${ }^{6}$, delle enclavi urbane o dei no man's land alla periferia delle città che i migranti si appropriano o che vengono loro concesse.

Queste procedure di aggiramento del divieto di mobilità hanno altresì fatto emergere un loro proprio settore d'attività: la politica di chiusura delle frontiere, generalizzando la restrizione

\footnotetext{
${ }^{6}$ Per alcune descrizioni dettagliate dell'organizzazione sociale dei ghetti di migranti, cf. Pian (2009), Laacher (2007).
} 
dei visti che vige dal 1990 (convenzione di Dublino), ha fatto della libertà di circolare un valore di scambioed ha fatto emergere un'economia del passaggio. Lungo le città di tappa di questi spostamenti si è visto svilupparsi tutto un settore economico che fornisce lavoro informale ad una moltitudine di attori sociali: passatori, intermediari, servizi di alloggio o di ristorazione per i viaggiatori.

Infine, tutte queste pratiche migranti e queste attività legate al transito si dispiegano in reti transnazionali, formali o informali, dove i migranti interagiscono con attori periferici rispetto al dispositivo di controllo, che sono gli organismi che l'accompagnano nel suo aspetto umanitario (HCR, IOM, Croce Rossa, Médecins du Monde), gli NGO e le associazioni locali, le chiese e le organizzazioni religiose (Caritas) e tutto un pullulare di organismi presenti nel "mercato del transito" che si posizionano relativamente all'assistenza ai migranti, alle campagne di dissuasione contro l'immigrazione clandestina, il reinserimento e la formazione, ecc. Questa dualità dei compiti di controllo e di assistenza può far pensare a ciò che Michel Agier descrive a proposito dei campi di rifugiati: le operazioni di polizia finalizzate a sorvegliare e controllare sono controbilanciate dagli interventi di coloro che nomina, riprendendo l'espressione di Bourdieu, "la mano sinistra dell'Impero" (Agier 2007).

Riguardo al secondo aspetto, queste mobilità presentano i tratti caratteristici di una individualizzazione delle migrazioni, che si manifesta attraverso delle strategie personali di accumulazione economica e di presa di distanza rispetto alle costrizioni dell'ambiente di origine. Tramite questa individualizzazione del senso della migrazione si esprime una richiesta di riconoscenza, in una situazione in cui partire appare come l'unico progetto di avvenire da parte di giovani uomini che la propria società d'origine condanna, a causa della loro incapacità a fornire sussidi all'economia familiare, ad una sorta di minorità sociale (Timéra 2001). Questo processo d'individualizzazione non è nuovo, il sociologo Abdelmalek Sayad (1977) mostrava come era già in germe negli anni 70 nell'immigrazione algerina. Ma le prove inerenti al superamento della frontiera lo hanno confortato, conferendogli una dimensione eroica. Diventata in senso proprio un combattimento per la vita o la morte ${ }^{7}$, la migrazione è ormai propizia all'esaltazione dei valori individualisti: contare su se stessi, avere il coraggio di cercare completamente da soli il proprio cammino nella vita.

A causa della lunghezza del viaggio, che a volte può estendersi su più anni nell'attesa dell'occasione favorevole, i migranti sono portati a circolare in una molteplicità di luoghi, lo spazio - come dicono - "dei paesi dove si gira": quelli dove si limitano a passare, quelli dove indugiano, quelli dove ormeggiano per dispiegare le loro circolazioni, quelli dove, eventualmente, si stabiliscono. È dunque nelle temporalità proprie al percorso migratorio stesso, nel progredire delle sue tappe, delle sue prove e delle sue opportunità, che si costruisce un'esperienza sociale specifica: quella che si trova condensata nella nozione di "avventura" e nei suoi derivati (il sostantivo "avventuriero", il verbo "avventurarsi"), largamente utilizzati

\footnotetext{
${ }^{7}$ Come è espresso in wolof dal grido di adunata dei giovani senegalesi per il superamento delle barriere di Ceuta: Barça mba barzakh (Barcellona o la morte).
} 
dai migranti per presentarsi nei racconti personali che fanno dei propri percorsi, nei diari che redigono, o sotto forma di espressioni collettive (blogs, lavori teatrali).

L'avventura è la figura rovesciata della categoria amministrativa del transito: una nozione emica, che totalizza un'esperienza della vita, la forma soggettivata di un insieme di condizioni sociali specifiche:

- il distaccarsi di questo tipo di mobilità dalle filiere migratorieprecostituite che organizzano le traiettorie di migrazione, come quelle dei Soninke verso la regione parigina. Le migrazioni dell'avventura si costruiscono come imprese individuali, se non addirittura solitarie, che non si appoggiano su catene migratorie collaudate da lungo tempo, in tal modo che i migranti dell'avventura sono anche quelli che aprono fronti pionieristici a nuovi itinerari migratori. Avventurarsi vuol dire partire da casa propria per avviare un percorso di cui si sa che non sarà lineare, come quello del lavoratore migrante che andava da un punto ad un altro, ma che consisterà nel "cercare la rotta".

- una disposizione di questi migranti rispetto alla migrazione molto differente rispetto a quella del lavoratore immigrato, espressa attraverso le nozioni di "fortuna" e di "destino" che ritornano frequentemente nei discorsi. Ciò che caratterizza innanzitutto la migrazione d'avventura è l'imprevedibilità della vita sociale e l'assenza di padronanza sull'orizzonte temporale dei progetti. Di modo che il senso stesso dell'avventura per ciascun individuo si forma in situazione, nella successione delle prove attraverso le quali si addomestica "la fortuna", questa divinità capricciosa degli avventurieri. Ciò che si esprime attraverso queste parole, la "fortuna", il "destino", è un'attitudine di accettazione del rischio implicato dal carattere azzardoso dell'impresa e della durezza delle prove, accettazione spesso sacralizzata dalla religione. L'avventuriero è nella mano di Dio. Si trovano costantemente frammisti nei discorsi raccolti i due elementi che caratterizzano la nozione di avventura quale l'analizza Simmel (2002): la forza conquistatrice (la capacità personale di sormontare gli ostacoli con il propriocoraggio, la propria astuzia, la propria intelligenza) e il dono gratuito che non si può forzare, che fa dipendere la riuscita dalla fortuna come elemento esterno a sé e incalcolabile. È questa disposizione che viene generalmente stigmatizzata come "incoscienza", "cecità", o "illusione" dai commentatori occidentali (che utilizzano a profusione il cliché dell'"eldorado dell'Europa", del "miraggio", della "follia suicidaria") per mettere in contrasto queste migrazioni con quelle ragionate e ragionevoli dell'epoca anteriore ${ }^{8}$.

- un'altra caratteristica di questa esperienza della migrazione è che rappresenta uno spaziotempo al di fuori della continuità della vita (Simmel 2002), un ritiro fuori dai modi normali dell'azione sociale. Nei racconti di questi migranti si trovano tutti gli elementi caratteristici dei riti di passaggio (Van Gennep 1909 \{1981\}, Turner 1969): la dimensione di prova, il mettere fra parentesi gli attributi di status e i diritti e doveri che vi sono legati, lo spirito di

\footnotetext{
${ }^{8} \mathrm{Si}$ noti che, contrariamente alla rappresentazione condiscendente dell'eldorado, non c'era nulla d'irrazionale, all'epoca della nostra inchiesta, nel cercare di raggiungere le zone dove si sapeva che c'era una richiesta di lavoro informale, come era il caso della Spagna fino alla crisi.
} 
cameratismo e di egualitarismo che trascende senza abolirle le distinzioni di rango (primogenito/cadetto, sposato/celibe, nobile/dipendente).

Non tutti gli avventurieri sono migranti di transito ${ }^{9}$ e non tutti i migranti in transito sono avventurieri. Per alcuni, il tentativo di passaggio clandestino rappresenta solo una strategia alternativa per raggiungere, con altri mezzi, gli stessi obbiettivi che gli immigrati dell'epoca anteriore potevano raggiungere tramite vie legali: in particolare quelli legati allo sviluppo economico della famiglia che ha fatto una colletta per pagare il viaggio e di cui sono gli emissari e i debitori.

Ma anche per questi la situazione di attesa finisce coll'allentare i legami familiari e coll'integrarli in una comunità di itineranza (Escoffier 2006). La migrazione, i suoi obbiettivi, i suoi progetti, si cancellano di fronte ai rischi del viaggio che pongono l'individuo nell'ambiguità fra stati passati e futuri: non è più un membro della società locale che ha lasciato, ma ancora non è (e forse non sarà mai) un immigrato.

Fra questi due stati, il percorso dell'avventuriero è un'erranza, ma di fatto un'erranza molto socializzata, che genera le sue proprie reti di solidarietà e di sociabilità, distinte dai legami obbligati di solidarietà fra "fratelli" (compatrioti o co-etnici) che possono sempre essere mobilizzati in un paese straniero. L'avventura moltiplica le occasioni di incontro, di scambi, di transazioni con sconosciuti, legami che la maggior parte delle volte sono transitori e senza indomani, legami deboli (Granoveter 1973) e tanto più efficaci per massimizzare e diversificare le informazioni utili (sulle "fregature" da evitare, le vie che è meglio abbandonare, gli indirizzi per un alloggio provvisorio, ecc.).

Se si vuol riassumere ciò che caratterizza queste mobilità si può notare:

- l'elevato grado di coinvolgimento dei migranti, la loro determinazione ad agire quali che siano i rischi, e la loro capacità d'organizzazione e di cooperazione in azioni congiunte e concertate come i passaggi in forza delle barriere.

- una solidarietà ben reale tra compagni uniti in una comunità di sventura, che si manifesta in particolar modo nel caso di decessi e malattie, malgrado le condizioni precarie e l'indigenza.

- la loro forte dimensione di ribellione, nutrita da un sentimento d'ingiustizia, l'ingiustizia che rappresenta l'assegnazione a residenza e il divieto di muoversi.

Questa dimensione di ribellione si esprime tramite sporadiche esplosioni di collera quali abbiamo potuto osservare, per esempio, durante le riunioni di dissuasione organizzate dagli organismi incaricati del controllo. Ma queste mobilità non si prestano affatto alla mobilitazione protestataria. Da una parte sono portate da un'ideologia fortemente

\footnotetext{
${ }^{9}$ Il termine di avventuriero è stato utilizzato a proposito degli "sapeurs" congolesi (Gandoulou 1984), dei
} trafficanti di diamanti (Bredeloup 2007), dei Ghettomen di Abidjan (De Latour 1999). 
individualista ("ad ognuno la sua chance") che non impedisce il mutuo riconoscimento fra la gente che condivide questa ideologia ed ha interessi in comune, ma confina l'azione collettiva a cooperazioni puntuali ed orientate verso uno scopo a breve termine (superamento delle barriere, organizzazione della vita nei ghetti). D'altra parte, quando emergono dei leader non possono imporsi come attori che contano se non allineandosi sulle agende e le linee d'azione degli organismi finanziatori o sul linguaggio militante delle associazioni di sostegno. Possono portare la parola dei "clandestini" solo se pagano il prezzo di un allontanamento dalle comprensioni condivise che rendono queste mobilità socialmente e moralmente legittime agli occhi dei loro attori: la migrazione come avventura, rivendicata come un'epopea eroica di colui che "cerca la sua chance"; come eredità di una tradizione che fa del soggiorno provvisorio in un universo straniero un rito di passaggio all'età adulta; o come dovere familiare che comanda di andare a cercare là dove si trovano le risorse che permettono di saldare il proprio debito sociale. Tutti questi significati contenuti nella parola avventura sono difficilmente traducibili in un lessico istituzionale o in parole d'ordine militanti. La sola parola d'ordine unificatrice è la libertà di circolazione che le istituzioni dispensatrici di sussidi non riconoscono e che le associazioni di sostegno mettono in avanti sul registro retorico, ma senza poterla tradurre in azioni mobilitanti concrete.

Nel caso dei migranti dell'avventura, ciò che si constata è la difficoltà nel tradurre la capacità d'azione collettiva spontanea, come quella che si manifesta nei ghetti, tramite un repertorio di azioni che sia al tempo stesso udibile da parte dei loro sostegni potenziali (a cominciare con gli organismi dispensatori di risorse che sono indispensabili per la sopravvivenza delle associazioni), e suscettibile d'essere fatto proprio dai migranti.

La migrazione irregolare è un tema eminentemente politico, ma paradossalmente il migrante irregolare non è un soggetto politico ${ }^{10}$. I migranti in transito vengono recepiti solo come delinquenti o vittime, non come soggetti, ma comeoggetti di pratiche di polizia o umanitarie. Colpisce constatare, in questo periodo d'intensa mediatizzazione e di emozione collettiva suscitate dai naufragi nel Mediterraneo, come le loro voci siano inaudibili. Ciò che viene offerto all'attenzione pubblica sono i servizi che fanno appello alla compassione per soggetti che soffrono, o narrano delle misure di polizia per fermarli nei loro tentativi di passaggio, focalizzate sulla punizione dei passatori, la cui figura costituisce più che mai "il cattivo per antonomasia" (Guiraudon 2008). Da una parte questo permette di connettere i registri opposti della repressione e della compassione: le attività di polizia che mirano i migranti colpevoli di frode possono simultaneamente essere viste come delle attività di protezione di vittime dei trafficanti; dall'altra questa focalizzazione sui passatori fa scordare la violenza di stato, come nel 2005 la deportazione da parte del Marocco di centinaia di migranti sub-sahariani verso l'Algeria, abbandonati nel deserto, o gli attacchi mortali contro i migranti sudanesi da parte della polizia egiziana.

\footnotetext{
${ }^{10}$ Contrariamente ai "sans papiers" in Francia, le cui rivendicazioni hanno potuto essere integrate nelle pratiche militanti della difesa dei lavoratori (con il sostegno della CGT) o del diritto all'educazione (con il sostegno del Réseau Education sans Frontières).
} 
Cionondimeno gli avvenimenti recenti mettono in luce una nuova configurazione dell'economia del passaggio.

Per i migranti dell'avventura, di cui abbiamo ritracciato qui l'esperienza migratoria, la traversata dal Marocco o dalla Mauritania verso la Spagna si faceva in un primo periodo imbarcandosi clandestinamente su barche da pesca industriale, poi più tardi su piccole imbarcazioni di fortuna, canotti pneumatici, pateras, piroghe, cayukos.

L'economia del passaggio poggiava di fatto su di un continuum tra i candidati al passaggio e quelli che li scortano, le cui attività erano più o meno lucrative in funzione delle risorse di cui disponevano: dalla persona al vertice, che compra o fa costruire le imbarcazioni, fino aicapi d'equipaggio (il capitano della piroga e il suo assistente) che beneficiano solo della gratuità del proprio passaggio giustificata dalla loro competenza di navigazione più o meno appurata e dal possesso di un GPS, passando per i "coxers" che riempiono le piroghe, e i diversi piccoli intermediari, affittuari di camere di passaggio, o immigrati stabiliti incaricati della prospezione presso le proprie comunità rispettive.

Quell'economia del passaggio faceva vivere un intero piccolo popolo di sensali e di intermediari che per la maggior parte vivevano nello stesso tessuto sociale e economico di quelli presso i quali riempivano queste funzioni.

Il circuito del passaggio funzionava infatti come un'agenzia di viaggio informale che, appoggiandosi su staffette da città a città, prendeva a carico la totalità del viaggio: dal convoglio in macchina fino al luogo d'imbarco, l'alloggio sul posto in attesa della partenza, le istruzioni date ai "viaggiatori", la costituzione della piroga. Poiché era demoltiplicata e informale da un'estremità all'altra, la filiera implicava in ciascuno dei suoi punti di connessione il rischio di essere vittima di pratiche disoneste e in effetti gli "imbrogli" vi erano per così dire ordinaria amministrazione. Per cui il problema principale che i candidati al passaggio si ponevano riguardo ai passatori era, come in fondo con qualunque operatore in un mercato, quello di distinguere fra le persone oneste e i mascalzoni.

Questa economia del passaggio ha conosciuto recentemente un cambiamento radicale di ordine di grandezza: con la chiusura delle vie anteriori, i migranti d'Africa subsahariana raggiungono sulla via libica l'afflusso dei rifugiati che fuggono situazioni di guerra civile o di violenza (Siria, Sudan, Eritrea, Somalia). Nello stesso tempo in cui si sviluppa ciò che Claire Rodier (2013) descrive come un "xénophobie business" (l'intensificazione e la privatizzazione dei dispositivi di sorveglianza e di ritenzione), il passaggio tende a professionalizzarsi come un'impresa del crimine organizzato, con un aumento considerevole dei rischi di morte e di violenza. Poiché se c'è qualcosa di costante è proprio che ogni inasprimento delle misure di controllo che chiude delle vie ne apre di nuove ancora più pericolose, ogni rinforzo delle misure di sorveglianza è un passo supplementare nella violenza. 
L'ossessione dell'invasione, che fa della violenza la caratteristica della mobilità dei nulla abbienti, pone non solo un problema agli immigrati e ai rifugiati che ne sono gli oggetti e le vittime, ma pone anche un problema di morale politica alle società europee, poiché mettono in causa il loro proprio modello di democrazia che concede un posto centrale al regime dei diritti dell'uomo.

La gestione politica e mediatica delle migrazioni nell'epoca attuale erge in maniera insidiosa una frontiera non solo sociale ma morale fra Noi e Loro. Il diritto alla mobilità tende a diventare l'asse intorno al quale si ridistribuisce una nuova forma di partizione dell'umanità, quella che separa quelli che hanno ogni legittimità nel circolare e quelli che sono costretti all'immobilità e i cui spostamenti possono solo essere fraudolenti, clandestini, illegali, in breve: immorali. Cosicché ci si può legittimamente chiedere se la nuova forma di gerarchizzazione mondiale che rappresenta l'accesso differenziato alla mobilità (Bauman 1998) altro non sia che la riattualizzazione, in un mondo globalizzato, della grande divisione antropologica che un tempo distribuiva l'umanità in civilizzati e non civilizzati.

\section{Bibliografia}

Agier M. (2007) Le chaos et les camps. Genèse du gouvernement humanitaire, in PaugamSerge (dir.) Repenser la solidarité, Paris, PUF, collection Le lien social, pp. 797-815.

Bauman, Z. (1998), Globalization. The Human Consequences, London, Polity Press \& Blackwell Publishers Ltd.

Bredeloup, S. (2007), La Diams 'pora du fleuve Sénégal, Toulouse, Presses Universitaires du Mirail.

De Latour, E. (1999), "Les ghettomen. Les gangs de rue à Abidjan et San Pedro", Actes de laRecherche en Sciences Sociales, $\mathrm{n}^{\circ}$ 129: 68-83.

Escoffier, C. (2006), “Communautés d'itinérance et savoir-circuler des transmigrant-e-s au Maghreb", Recueil Alexandries, Collections Etudes, juin, url di riferimento: http://www.reseau-terra.eu/article511.html

Gandoulou, J.D. (1984), Entre Paris et Bacongo, Paris, Centre Georges Pompidou. 
Georgi, F. (2010), 'For the Benefit of Some: The International Organization for Migration and its Global Migration Management”, in: Geiger M. \& Pécoud A.: The Politics of International Migration Management, Palgrave Macmillan: 45-72

Granovetter, M.S. (1973), "The Strength of Weak Ties", American Journal of Sociology, Volume 78, Issue 6 (May): 1360-1380.

Guiraudon, V. (2008), "Une lutte contre les passeurs qui se retourne contre les victimes", in: (curato da De Claire Rodier \& Emmanuel Terray), Immigration: fantasmes et réalités, Paris, La Découverte.

Laacher, S. (2007), Le peuple des clandestins, Paris, Calmann-Lévy.

LeHouérou, F. (2007), "Migrations Sud-Sud: Les Circulations Contrariées des Migrants vers le Monde Arabe", REMMM, n¹19-120, novembre: 9-21.

Pian, A. (2009), Aux nouvelles frontières de l'Europe. L'aventure incertaine des Sénégalais au Maroc, Paris, La Dispute.

Rodier, C. (2013), Xénophobie Business. A quoi servent les contrôles migratoires, Paris, La Découverte.

Sayad, A. (1977), "Les trois âges de l'émigration algérienne en France", Actes de la Recherche en Sciences Sociales, $\mathrm{n}^{\circ}$ 15: 59-79.

Simmel, G. (2002), Philosophie de l'aventure, L'Arche.

Streiff-Fénart J. \& Segatti A. (2011), The Challenge of the Threshold. Border Closures and Migration Movements in Africa, Maryland, Lexington Books.

Timéra, M. (2001), “Les migrations des jeunes Sahéliens: affirmation de soi et émancipation", Autrepart, $\mathrm{N}^{\circ} 18: 37-49$.

Turner, V.W. (1969), The Ritual Process: Structure and Anti-Structure, Penguin.

VanGennep, A. (1981), Les rites de passage, Paris, Picard. 